\title{
Common names for Australian ants (Hymenoptera: Formicidae)
}

\author{
Alan N Andersen \\ CSIRO Sustainable Ecosystems, Tropical Ecosystems Research Centre, PMB 44, Winnellie, NT 0822, Australia.
}

\begin{abstract}
Most insects do not have common names, and this is a significant barrier to public interest in them, and to their study by non-specialists. This holds for even highly familiar insect groups such as ants. Here, I propose common names for all major native Australian ant genera and species-groups, as well as for many of the most abundant and distinctive species. Sixty-two genera, 142 species-groups and 50 species are given names. The naming system closely follows taxonomic structure; typically a genus is given a general common name, under which species-group and species names are nested.
\end{abstract}

Key words ant species, communicating entomology, species-groups, taxonomic nomenclature.

\section{INTRODUCTION}

Common names are powerful aids for the popular communication of information about plant and animal species. Such names use familiar and easily remembered words, in contrast to the taxonomic nomenclature that is so daunting for most people without formal scientific training. All higher-profile vertebrates and vascular plants have widely accepted common names. These increase the accessibility of these species to a wide public audience, and promote interest in them. In contrast, the vast majority of insects and other arthropods have no common name beyond the ordinal level, unless they are important pests (Naumann 1993). Relatively high-profile groups such as butterflies are notable exceptions (Miller 1992; Braby 2000), and the availability of common names helps attract public interest in them (Braby et al. 1997).

For most insect groups, however, a lack of common names consigns them to public obscurity. It also puts them in the 'too-hard basket' from the perspective of most amateur naturalists and indeed, many researchers with a background in traditional wildlife biology. In short, a lack of common names would appear to be one reason why the study of most insect groups remains largely within the exclusive domain of specialist entomologists. Given Australia's relatively small number of specialist entomologists, particularly when measured against its 'megadiverse' insect fauna, anything that promotes public interest in insects should be encouraged.

Ants are one of the most familiar groups of insects throughout the world, and are arguably the most important faunal group in the Australian environment (Matthews \& Kitching 1984). They are widely used as biological indicators of ecosystem health (Majer 1983; Andersen 1990). In the face of such prominence, there is a remarkable lack of common names for ants. To most Australians, there are just three types of ant: bull ants (typically referring to species of Myrmecia spp.), sugar ants (usually Camponotus spp.), and

Email: alan.andersen@csiro.au 'little black ones' (the remaining several thousand Australian species). Here, I attempt to redress this situation by proposing common names for all major native Australian ant genera and species-groups, as well as for many abundant and distinctive species.

\section{PROPOSED ANT COMMON NAMES}

Proposed common names, and explanations for them, for 62 genera, 142 species-groups and 50 species of Australian ants are presented in Appendix I, Table A1. Species-groups follow Andersen (1991a, 2000), and authorities for genera and species are listed in Taylor and Brown (1985) and Shattuck (1999).

The system of common names closely follows taxonomic structure. Each genus is given a general common name, under which species-group and species names are nested. The advantage of this approach is that the common names reflect higher taxa and therefore indicate systematic relationships (Miller 1992; Braby et al. 1997). A disadvantage is that species-level names are composed of multiple words, which can be rather cumbersome. However, this would seem inevitable when dealing with highly diverse genera containing poorly known species, with no history of common names. Moreover, names at the species-group or genus level will suffice for most popular communication about ants, particularly given that most Australian species do not even have scientific names.

\section{EXISTING ANT COMMON NAMES}

The online catalogue of Australian Insect Common Names (AICN) (CSIRO 2001) lists common names for 15 native Australian ant species and three genera. In many cases the names proposed here are consistent with these (Table 1). The following are cases where I believe existing AICN names are inappropriate: 
Table 1 Existing common names for native Australian ants as catalogued in Australian Insect Common Names (CSIRO, 2001), with new common names proposed here

\begin{tabular}{lll}
\hline Scientific name & AICN common name & Proposed common name \\
\hline Aenictus spp. & Army ants & Lesser army ants \\
Aphaenogaster pythia & Funnel ant & Funnel ant \\
Camponotus consobrinus & Sugar ant & Banded sugar ant \\
Crematogaster laeviceps chasei & Cocktail ant & Valentine ant \\
Doleromyrma darwiniana & Brown house ant & None \\
Iridomyrmex purpureus & Meat ant & Southern meat ant \\
Iridomyrmex spp. & Meat ants & Tyrant ants \\
Melophorus bagoti & Honeypot ant & Bagot's furnace ant \\
Myrmecia brevinoda & Giant bulldog ant & Giant bull ant \\
Nothomyrmecia macrops & Dinosaur ant & Dinosaur ant \\
Ochetellus glaber & Black house ant & Black house ant \\
Oecophylla smaragdina & Green tree ant, or weaver ant & Green tree ant \\
Onychomyrmex spp. & Army ants & False army ants \\
Pheidole ampla, P. anthracina & Seedharvesting ants & Big-headed ants \\
Polyrhachis macropus & Mulga ant & Mulga spiny ant \\
Polyrhachis ornata & Golden ant & Superb spiny ant \\
Rhytidoponera spp. & Greenhead ants & Pony ants \\
\hline
\end{tabular}

- Crematogaster laeviceps chasei (AICN name: cocktail ant). The term 'cocktail ant' is more appropriately applied to species with a stronger aroma, such as those of Anonychomyrma and Papyrius.

- Doleromyrma darwiniana (brown house ant). This species is not particularly associated with human settlement. Several other common pest ants in houses are similarly 'brown'.

- Iridomyrmex spp. (meat ants). The term meat ant should be reserved for the distinctive purpureus group of Iridomyrmex, rather than the entire genus.

- Melophorus bagoti (honeypot ant). The extent to which this species maintains repletes ('honeypots') is unclear, and the term 'honeypot ant' is more commonly applied to the highly familiar Camponotus inflatus.

- Pheidole spp. (seed-harvesting ants). The extent of granivory varies greatly within Pheidole (Andersen 1991b). Species of the hartmeyeri group are specialist harvesters (Table 1), but some other groups do not appear to be at all granivorous.

- Polyrhachis ornata (golden ant). Species from a range of genera are covered with golden pubescence, and such pubescence occurs independently within several subgenera of Polyrhachis. The term 'golden' is therefore not very informative.

- Rhytidoponera spp. (greenhead ants). The term 'greenhead ant' is commonly applied to a species of the metallica group in south-eastern Queensland, but a green head is not at all characteristic of the genus.

\section{ACKNOWLEDGEMENTS}

I am extremely grateful to Andy Chapman (CSIRO Sustainable Ecosystems), Brian Heterick (Curtin University of Technology), Maryanne McKaige (Northern Territory University),
Ian Naumann (Department of Agriculture, Fisheries and Forestry, Australia), Tim New (La Trobe University) and Ms Val Speedie (Undara Lava Lodge) for their comments on the manuscript and suggestions for common names.

\section{REFERENCES}

Agosti D. 1997. Two new engimatic Melophorus species (Hymenoptera: Formicidae) from Australia. Journal of the New York Entomological Society 105, 161-169.

Andersen AN. 1990. The use of ant communities to evaluate change in Australian terrestrial ecosystems: a review and a recipe. Proceedings of the Ecological Society of Australia 16, 347-357.

Andersen AN. 1991a. The Ants of Southern Australia: A Guide to the Bassian Fauna. CSIRO Press, Melbourne, Australia.

Andersen AN. 1991b. Seed-harvesting by ants in Australia. In: Ant-Plant Interactions (eds CR Huxley \& DF Cutler) pp. 493-503. Oxford University Press, Oxford, UK.

Andersen AN. 2000. The Ants of Northern Australia: a Guide to the Monsoonal Fauna. CSIRO Publishing, Melbourne, Australia.

Bolton B. 1977. The ant tribe Tetramoriini (Hymenoptera: Formicidae). The genus Tetramorium Mayr in the Oriental and Indo-Australian regions, and in Australia. Bulletin of the British Museum (Natural History), Entomology 36, 67-151.

Braby MF. 2000. Butterflies of Australia: Their Identification, Biology and Distribution. CSIRO Publishing, Melbourne, Australia.

Braby MF, Atkins AF, Dunn KL, Woodger TA \& Quick WNB. 1997. A provisional list of common names for Australian butterflies. Australian Journal of Entomology 36, 197-212.

Clark J. 1941. Australian Formicidae. Notes and new species. Memoirs of the National Museum of Victoria 12, 71-94.

CSIRO. 2001. Australian Insect Common Names. CSIRO Publishing, Collingwood, Australia. http://www.ento.csiro.au/aicn/

Gotwald WH Jr. 1995. Army Ants: The Biology of Social Predation. Comstock Publishing Associates, Cornell University Press, Ithaca, New York, USA.

Majer JD. 1983. Ants: bioindicators of mine site rehabilitation, land use and land conservation. Environmental Management 7, $375-383$.

Matthews EG \& Kitching RL. 1984. Insect Ecology. University of Queensland Press, Brisbane, Australia. 
McAreavey JJ. 1947. New species of the genera Prolasius Forel and Melophorus Lubbock (Hymenoptera: Formicidae). Memoirs of the National Museum of Victoria 15, 7-27.

McArthur AJ \& Adams M. 1996. A morphological and molecular revision of the Camponotus nigriceps group (Hymenoptera: Formicidae) from Australia. Invertebrate Taxonomy 10, 1-46.

McArthur AJ \& Shattuck SO. 2001. A taxonomic revision of the Camponotus macrocephalus species group (Hymenoptera: Formicidae) in Australia. Transactions of the Royal Society of South Australia 125, 25-43.

Miller JY. 1992. The Common Names of North American Butterflies. Smithsonian Institution Press, Washington DC, USA.

Morton SR \& Christian KA. 1994. Ecological observations on the spinifex ant, Ochetellus flavipes (Kirby) (Hymenoptera: Formicidae), of Australia's northern arid zone. Journal of the Australian Entomological Society 33, 309-316.

Naumann I. 1993. CSIRO Handbook of Australian Insect Names. Common and Scientific Names for Insects and Allied Organisms of Economic and Environmental Importance, 6th edition. CSIRO, Melbourne, Australia.

Nielsen MG. 2000. Distribution of the ant (Hymenoptera: Formicidae) fauna in the canopy of the mangrove tree Sonneratia alba J. Smith in northern Australia. Australian Journal of Entomology 39, 275-279.
Ogata K \& Taylor RW. 1991. Ants of the genus Myrmecia Fabricius: a preliminary review and key to the named species (Hymenoptera: Formicidae: Myrmeciinae). Journal of Natural History 25, $1623-1673$.

Peeters C \& Higashi S. 1989. Reproductive dominance controlled by mutilation in the queenless ant Diacamma australe. Naturwissenschaften 76, 177-180.

Shattuck SO. 1993. Revision of the Iridomyrmex purpureus speciesgroup (Hymenoptera: Formicidae). Invertebrate Taxonomy $\mathbf{7}$, 113-149.

Shattuck SO. 1999. Australian Ants: Their Biology and Identification. CSIRO Publishing, Collingwood, Australia.

Taylor RW. 1978. Nothomyrmecia macrops: a living-fossil ant rediscovered. Science 201, 979-985.

Taylor RW. 1991. Nomenclature and distribution of some Australian ants of the Myrmicinae (Hymenoptera: Formicidae). Memoirs of the Queensland Museum 30, 599-614.

Taylor RW \& Brown DR. 1985. Formicoidea. In: Zoological Catalogue of Australia, Vol. 2. Hymenoptera, Vespoidea and Sphecoidea (ed. DW Walton) pp. 1-149. Australian Government Publishing Service, Canberra, Australia.

Accepted for publication 19 April 2002.

\section{APPENDIX I}

Table AI Proposed common names for major Australian ant genera and species-groups, and some abundant and distinctive species

\begin{tabular}{|c|c|c|}
\hline Scientific name & Common name & Explanation \\
\hline \multicolumn{3}{|l|}{ Subfamily Aenictinae } \\
\hline Aenictus & Lesser army ants & $\begin{array}{l}\text { True army ants, but small, monomorphic and without the nomadic } \\
\text { lifestyle of their famous cousins (Gotwald 1995) }\end{array}$ \\
\hline A. atratus & Black lesser army ant & Colour black \\
\hline A. ceylonicus group & Yellow lesser army ants & Colour yellowish \\
\hline \multicolumn{3}{|c|}{ Subfamily Cerapachyinae } \\
\hline Cerapachys & Cannibal ants & As with the related Sphinctomyrmex, specialist ant brood-raiders \\
\hline C. brevis group & Little cannibal ants & Small species \\
\hline C. clarki group & Speckled cannibal ants & Dorsum of petiolar node often punctate \\
\hline C. edentatus group & Blind cannibal ants & Eyes absent \\
\hline C. fervidus group & Two-lined cannibal ants & Dorsolateral margins of trunk with carinae running their entire length \\
\hline C. longitarsus group & Topless cannibal ants & Trunk without distinct dorsal face \\
\hline C. singularis group & Angle-headed cannibal ants & Head with lateral carinae \\
\hline Sphinctomyrmex & Sausage cannibal ants & See Cerapachys; gaster elongate and serially constricted \\
\hline \multicolumn{3}{|c|}{ Subfamily Dolichoderinae } \\
\hline Anonychomyrma & Black cocktail ants & Strongly aromatic; uniformly black \\
\hline A. biconvexa & Forest black cocktail ant & Occurs in wetter forests \\
\hline A. gilberti & Golden black cocktail ant & Golden pubescence \\
\hline Dolichoderus & Dolly ants & Derived from genus name \\
\hline D. australis group & Soft dolly ants & Integument relatively thin and feebly sculptured \\
\hline D. doriae group & Double-spined dolly ants & Pronotum and propodeum each with a pair of spines \\
\hline D. reflexus group & Shark-finned dolly ants & Propodeum raised and reflexed backwards \\
\hline D. scrobiculatus & Northern shark-finned dolly ant & Most northerly species of the group \\
\hline D. scabridus group & Spiny dolly ants & Propodeum with a pair of spines \\
\hline Froggattella & Froglet ants & Modified from genus name \\
\hline F. kirbii & Common froglet ant & Most common and widely distributed of the two species \\
\hline
\end{tabular}




\begin{tabular}{|c|c|c|}
\hline Scientific name & Common name & Explanation \\
\hline Iridomyrmex & Tyrant ants & Behaviourally dominant and highly aggressive \\
\hline I. agilis group & Agile tyrant ants & Derived from species-group name \\
\hline I. anceps group & Tropical tyrant ants & Tropical distribution \\
\hline I. bicknelli group & Dome-headed tyrant ants & Posterior of head characteristically dome-shaped \\
\hline I. calvus group & Calvus tyrant ants & Derived from species-group name \\
\hline I. conifer group & Coned tyrant ants & Conical propodeum \\
\hline I. cyaneus group & Blue tyrant ants & Integument with bluish iridescence \\
\hline I. gracilis group & Gracile tyrant ants & Derived from species-group name \\
\hline I. mattiroloi group & Little tyrant ants & Smallest of all Iridomyrmex \\
\hline I. pallidus group & Pale tyrant ants & Colour yellowish \\
\hline I. purpureus group & Meat ants & Established common name (Shattuck 1993) \\
\hline I. greensladei & Greenslade's meat ant & Derived from species name \\
\hline I. lividus & Blue meat ant & Integument with blue iridescence \\
\hline I. purpureus & Southern meat ant & Most common species in south-eastern Australia \\
\hline I. reburrus & Bearded meat ant & Sides of head with short hairs \\
\hline I. sanguineus & Northern meat ant & Most common species in northern Australia \\
\hline I. viridiaeneus & Centralian meat ant & Most common species in central Australia \\
\hline I. rufoinclinus group & Northern bearded tyrant ants & $\begin{array}{l}\text { As with closely related viridigaster group, underside of head with } \\
\text { numerous long, curved hairs; occurs in northern arid zone }\end{array}$ \\
\hline I. rufoniger group & Tufted tyrant ants & Pronotum with tuft of hairs \\
\hline I. suchieri group & Flat-backed tyrant ants & Flattened propodeum \\
\hline I. vicina group & Southern tyrant ants & Occurring in cool-temperate Australia \\
\hline I. viridigaster group & Southern bearded tyrant ants & See rufoinclinus group; occurs in southern arid zone \\
\hline Leptomyrmex & Spider ants & Exceptionally long legs give them a spider-like appearance \\
\hline L. erythrocephalus & Red-headed spider ant & Uniformly black except for contrasting red head \\
\hline \multicolumn{3}{|l|}{ Ochetellus } \\
\hline O. flavipes & Spinifex ant & $\begin{array}{l}\text { Established common name, because of association with species of Triodia } \\
\text { (Morton \& Christian 1994) }\end{array}$ \\
\hline O. glaber group & Black house ants & Established common name \\
\hline Papyrius & Red cocktail ants & Strongly aromatic; uniformly reddish brown \\
\hline Tapinoma & Pedicel ants & As in Technomyrmex, propodeum without distinct node \\
\hline T. minutum group & Dwarf pedicel ants & Tiny size \\
\hline Technomyrmex & Tropical pedicel ants & See Tapinoma; occurring primarily in the tropics \\
\hline Turneria & Turner ants & Derived from genus name \\
\hline \multicolumn{3}{|l|}{ Subfamily Formicinae } \\
\hline Acropyga & Root-aphid ants & Tend root aphids for honeydew \\
\hline Calomyrmex & Beauty ants & English translation of genus name \\
\hline C. impavidus & Black beauty ant & Uniformly black \\
\hline C. purpureus & Speckled beauty ant & Trunk densely punctate \\
\hline C. splendidus & Bauble beauty ant & Iridescent bauble-like gaster \\
\hline Camponotus & Sugar ants & Established common name \\
\hline C. anderseni & Mangrove plug ant & $\begin{array}{l}\text { As with species of the gasseri, janeti, macrocephalus, reticulatus and } \\
\text { vitreus groups, major workers phragmotic, using their heads to plug } \\
\text { nest entrances (McArthur \& Shattuck 2001). Nests exclusively in } \\
\text { mangroves (Nielsen 2000) }\end{array}$ \\
\hline C. aurocinctus group & Golden bearded sugar ants & $\begin{array}{l}\text { Along with the related ceriseipes, denticulatus and terebrans groups, has } \\
\text { a tuft of J-shaped hairs on the gula. Species of the aurocinctus group } \\
\text { characteristically have a strikingly golden gaster }\end{array}$ \\
\hline C. ceriseipes group & Southern bearded sugar ants & See aurocinctus group. Occurs primarily in the southern semi-arid zone \\
\hline
\end{tabular}




\begin{tabular}{|c|c|c|}
\hline Scientific name & Common name & Explanation \\
\hline C. claripes group & Pale-legged sugar ants & Pale legged \\
\hline C. denticulatus group & Money-box sugar ants & See aurocinctus group. Nests have a slit-shaped entrance \\
\hline C. discors group & Yellow disc sugar ants & As in evae group, trunk rounded in profile, disc-like; colour yellowish \\
\hline C. ephippium group & Jumbuck sugar ants & Minor workers with angular, sheep-like heads \\
\hline C. evae group & Black disc sugar ants & See discors group; colour black \\
\hline C. gasseri group & Southern plug ants & See $C$. anderseni. Only 'plug ants' with a southern distribution \\
\hline C. innexus group & Antarctic sugar ants & $\begin{array}{l}\text { One of the few species-groups centred on cool-temperate southern } \\
\text { Australia }\end{array}$ \\
\hline C. intrepidus group & Flumed sugar ants & Nests often with chimney-like entrances \\
\hline C. agilis & Agile flumed sugar ant & From species name \\
\hline C. bendigensis & Red-legged flumed sugar ant & Blackish body with contrasting red legs \\
\hline C. intrepidus & Coastal flumed sugar ant & Restricted to coastal south-eastern Australia \\
\hline C. molossus & Western flumed sugar ant & Only species occurring in Western Australia \\
\hline C. piliventris & Inland flumed sugar ant & Widespread in inland south-eastern Australia \\
\hline C. suffusus & Golden flumed sugar ant & Gaster with golden pubescence \\
\hline C. janeti group & Janet's plug ants & See $C$. anderseni. From species-group name \\
\hline C. macrocephalus group & Rectangle plug ants & See $C$. anderseni. Rectangular trunk \\
\hline C. howensis & $\begin{array}{l}\text { Lord Howe Island rectangle } \\
\text { plug ant }\end{array}$ & Endemic to Lord Howe Island \\
\hline C. macrocephalus & Southern rectangle plug ant & Occurring in south-eastern Australia \\
\hline C. minimus group & Pygmy sugar ants & Very small for Camponotus \\
\hline C. nigriceps group & Common sugar ants & $\begin{array}{l}\text { Most conspicuous species-group in southern Australia (McArthur \& } \\
\text { Adams 1996) }\end{array}$ \\
\hline C. consobrinus & Banded sugar ant & First gastric segment orange, contrasting with remaining segments \\
\hline C. nigriceps & Black-headed sugar ant & From species name \\
\hline C. prostans & Western sugar ant & Restricted to south-western Australia \\
\hline C. nigroaeneus group & Black sugar ants & Most common species are uniformly black \\
\hline C. aeneopilosus & Golden black sugar ant & Gaster with striking golden pubescence \\
\hline C. amperei & Mallee black sugar ant & Widespread in mallee habitats of south-eastern Australia \\
\hline C. inflatus & Australian honey-pot ant & Australia's best-known honey-pot ant \\
\hline C. nigroaeneus & Southern black sugar ant & Restricted to mesic south-eastern Australia \\
\hline C. novaehollandiae group & Northern sugar ants & Most common Camponotus in northern Australia \\
\hline C. pellax group & Silver sugar ants & Typically with silvery pubescence \\
\hline C. reticulatus group & Netted plug ants & See $C$. anderseni. From species-group name \\
\hline C. rubiginosus group & Coconut sugar ants & Strong coconut smell when crushed \\
\hline C. subnitidus group & Aerial sugar ants & Antennae glabrous and exceptionally long \\
\hline C. terebrans group & Brown bearded sugar ants & See aurocinctus group. Colour brownish \\
\hline C. vitreus group & Northern plug ants & See $C$. anderseni. Tropical distribution \\
\hline C. vitreus & Common northern plug ant & By far most common species \\
\hline C. whitei group & Armoured sugar ants & Integument thick and heavily sculptured \\
\hline C. whitei & Common armoured sugar ant & Most common and widely distributed species of group \\
\hline C. group A (Andersen 2000) & Northern bearded sugar ants & See aurocinctus group. Occurs primarily in the western monsoonal tropics \\
\hline Melophorus & Furnace ants & Exceptionally thermophilic \\
\hline M. aeneovirens group & Giant beaked furnace ants & $\begin{array}{l}\text { As in related froggatti group and groups A and B, clypeus projecting over } \\
\text { base of mandibles. Very large ants }\end{array}$ \\
\hline M. anderseni group & Northern robber furnace ants & $\begin{array}{l}\text { As with species of the related fulvihirtus group, these ants 'rob' brood } \\
\text { from the nests of meat ants; occurring in the northern arid zone (Agosti } \\
\text { 1997) }\end{array}$ \\
\hline M. bruneus group & Brown furnace ants & From species-group name \\
\hline
\end{tabular}




\begin{tabular}{|c|c|c|}
\hline Scientific name & Common name & Explanation \\
\hline M. fieldi group & Field furnace ants & From species-group name \\
\hline M. froggatti group & Froggatt's beaked furnace ants & See aeneovirens group \\
\hline M. fulvihirtus group & Southern robber furnace ants & See anderseni group; occurs in southern arid zone (Clark 1941) \\
\hline M. hirsutus group & Barrel furnace ants & Trunk barrel-shaped \\
\hline M. iridescens group & Racing furnace ants & Long-legged and particularly fast-moving \\
\hline M. bagoti & Bagot's furnace ant & From species name \\
\hline M. mjobergi group & Pygmy furnace ants & Very small species \\
\hline M. pillipes group & Bottle-brush furnace ants & Tibiae clothed with long erect hairs \\
\hline M. potteri group & Bulldozer furnace ants & $\begin{array}{l}\text { Stout species with large mandibles, giving them the appearance of } \\
\text { miniature bulldozers; specialist predators of termites (McAreavey } \\
\text { 1947) }\end{array}$ \\
\hline M. wheeleri group & Harvester furnace ants & Specialist seed-harvesters \\
\hline$M$. group A (Andersen 2000) & Gracile beaked furnace ants & See aeneovirens group. Slim trunk and long legs \\
\hline M. group B (Andersen 2000) & Beaded beaked furnace ants & See aeneovirens group. Antennae with bead-like segments \\
\hline$M$. group C (Andersen 2000) & Silver furnace ants & Covered with silver pubescence \\
\hline$M$. group D (Andersen 2000) & Robust furnace ants & Trunk and legs relatively short and stout \\
\hline M. group E (Andersen 2000) & Rough furnace ants & Integument conspicuously sculptured \\
\hline$M$. group F (Andersen 2000) & Mystery furnace ants & Taxonomic relationships with other groups a mystery! \\
\hline Myrmecorhynchus & Possum ants & Arboreal \\
\hline Notoncus & Epaulet ants & Prominent pronotal shoulders \\
\hline N. ectatommoides group & Pronged epaulet ants & Pronged metanotal projection \\
\hline N. enormis group & Bulbous epaulet ants & Bulbous metanotal projection \\
\hline N. gilberti group & Smooth epaulet ants & Integument almost entirely smooth and shiny \\
\hline N. hickmani group & Yellow epaulet ants & Characteristically pale in colour \\
\hline N. spinisquamus group & Giant epaulet ants & Largest of all Notoncus \\
\hline Notostigma & False sugar ants & Camponotus-like \\
\hline Oecophylla smaragdina & Green tree ant & $\begin{array}{l}\text { Established common name in northern Australia, where local populations } \\
\text { have a strikingly green gaster }\end{array}$ \\
\hline Opisthopsis & Strobe ants & Jerky motion \\
\hline O. diadematus group & Black-capped strobe ants & Posterior part of head black \\
\hline O. haddoni & Savanna strobe ant & Most common species in monsoonal zone \\
\hline O. major & Tufted strobe ant & Pronotum with tuft of hairs \\
\hline O. pictus & Painted strobe ant & From species name \\
\hline O. rufithorax & Black-headed strobe ant & Head entirely black \\
\hline O. rufoniger & Red-headed strobe ant & Head entirely red \\
\hline Paratrechina & Parrot ants & Derived phonetically from genus name \\
\hline P. minutula group & Baby parrot ants & Very small \\
\hline P. obscura group & Swamp parrot ants & Characteristic of fringes of wetlands and other waterlogged habitats \\
\hline P. vaga group & Forest parrot ants & Widespread in forests and woodlands \\
\hline Polyrhachis & Spiny ants & Trunk and petiole characteristically spiny \\
\hline subgenus Campomyrma & No general common name & Extremely variable morphologically \\
\hline P. femorata group & Broad-nosed spiny ants & Frontal carinae very widely separated \\
\hline P. femorata & Southern broad-nosed spiny ant & Occurring in south-eastern Australia \\
\hline P. flavibasis & Northern broad-nosed spiny ant & Occurring in north-eastern Australia \\
\hline P. gravis group & Gravis spiny ants & From species name \\
\hline P. pseudothrinax & Unicorn spiny ant & Petiolar node with single, long medial spine \\
\hline$P$. hirsuta group & Hairy spiny ants & From species-group name \\
\hline P. inconspicua group & Little spiny ants & Relatively small size \\
\hline
\end{tabular}




\begin{tabular}{|c|c|c|}
\hline Scientific name & Common name & Explanation \\
\hline P. macropus group & Mulga spiny ants & Characteristic of mulga habitats \\
\hline P. micans group & False devil spiny ants & Myrma-like petiole \\
\hline P. patiens group & Toothed spiny ants & Propodeum with teeth rather than spines \\
\hline P. schwiedlandi group & Desert spiny ants & Major group in arid zone \\
\hline P. sidnica group & Antarctic spiny ants & Distribution centred on the cool-temperate zone \\
\hline subgenus Cyrtomyrma & Dome-backed spiny ants & Trunk dome-shaped \\
\hline subgenus Chariomyrma & Savanna spiny ants & Most species occur in monsoonal zone \\
\hline subgenus Hagiomyrma & Elegant spiny ants & Elegant appearance \\
\hline subgenus Hedomyrma & Superb spiny ants & Superb appearance \\
\hline subgenus Myrma & Devil spiny ants & Pitchfork-like petiole \\
\hline subgenus Myrmhopla & Topless spiny ants & In most species, trunk without a distinct dorsal face \\
\hline subgenus Myrmothrinax & Treble spiny ants & Petiole with three spines \\
\hline subgenus Polyrhachis & Hooked spiny ants & Massive, hook-like spines \\
\hline Prolasius & Mistral ants & Characteristic of cold habitats \\
\hline P. bruneus group & Brown mistral ants & Colour brownish \\
\hline P. nitidissimus group & Black mistral ants & Colour black \\
\hline$P$. pallidus group & Yellow mistral ants & Colour yellowish \\
\hline Pseudonotoncus & Spiny epaulet ants & Notoncus-like, but with propodeal and petiolar spines \\
\hline Stigmacros & Snuggle-pot ants & Small, 'cute'-looking and uniquely Australian \\
\hline subgenus Campostigmacros & Flat snuggle-pot ants & Trunk dorsally flattened \\
\hline subgenus Chariostigmacros & Speckled snuggle-pot ants & Often densely punctate \\
\hline subgenus Cyrtostigmacros & Bumpy snuggle-pot ants & Conspicuous metanotal tubercles \\
\hline subgenus Hagiostigmacros & Spiny snuggle-pot ants & Prominent propodeal and petiolar spines \\
\hline subgenus Stigmacros & Baby snuggle-pot ants & Smallest species \\
\hline \multicolumn{3}{|l|}{ Subfamily Leptanillinae } \\
\hline Leptanilla & Phantom army ants & $\begin{array}{l}\text { Subterranean, army ant-like species, with several known only from alates } \\
\text { collected at lights }\end{array}$ \\
\hline \multicolumn{3}{|l|}{ Subfamily Myrmeciinae } \\
\hline Myrmecia & Bull ants & Established common name (Ogata \& Taylor 1991) \\
\hline M. aberrans group & Wide-jawed bull ants & Mandibles broadly triangular \\
\hline M. gulosa group & Giant bull ants & Extremely large species \\
\hline M. mandibularis group & Toothless bull ants & Shaft of mandibles without teeth \\
\hline M. pilosula group & Jumping jacks & Established common name, because of distinctive jumping motion \\
\hline M. tepperi group & Buck-toothed bull ants & Asymmetrical mandibular teeth \\
\hline M. urens group & Baby bull ants & Smallest of all Myrmecia \\
\hline \multicolumn{3}{|l|}{ Subfamily Myrmicinae } \\
\hline Adlerzia & Thumbelina ants & 'Thumb print' sculpturing on pronotal dorsum \\
\hline Aphaenogaster & Funnel ants & Established common name; nest entrances with funnel-like craters \\
\hline A. barbigula group & Desert funnel ants & Occurring in southern semi-arid zone \\
\hline A. longiceps group & Forest funnel ants & Occurring in wetter habitats \\
\hline Colobostruma & Fierce gremlin ants & $\begin{array}{l}\text { Along with the related dacetines Epopostruma and Mesostruma, having a } \\
\text { peculiar 'alien'-like appearance. Species of Colobostruma have a rather } \\
\text { fierce expression }\end{array}$ \\
\hline Crematogaster & Valentine ants & Heart-shaped gaster \\
\hline C. laeviceps group & Common valentine ants & Most common species \\
\hline C. queenslandica group & Little valentine ants & Smallest species \\
\hline Epopostruma & Snappy gremlin ants & See Colobostruma; elongate, trap-jaw mandibles \\
\hline Lordomyrma & Royal ants & Derived from 'Lord', and morphologically deserving of such a title! \\
\hline
\end{tabular}




\begin{tabular}{|c|c|c|}
\hline Scientific name & Common name & Explanation \\
\hline Meranoplus & Shield ants & Dorsal surface of trunk expanded to form a shield-like plate \\
\hline M. dimidiatus group & Box shield ants & Trunk box-like, with dorsal flanges feebly developed \\
\hline M. diversus group & Harvester shield ants & Specialist granivores \\
\hline M. fenestratus group & Holy shield ants & Dorsal flanges with translucent 'windows' \\
\hline M. froggatti group & False turtle ants & Apparently related to testudineus group, but with flanges not so developed \\
\hline M. hirsutus group & Jungle shield ants & Major group of tropical rainforest \\
\hline M. mjobergi group & Chocolate shield ants & Integument invariably dark-brown and smooth \\
\hline M. testudineus group & Turtle ants & Dorsal flanges remarkably extensive \\
\hline Mesostruma & Solemn gremlin ants & See Colobostruma; solemn expression \\
\hline Metapone & Termite-gallery ants & Highly specialised to live in termite galleries (Taylor 1991) \\
\hline Monomorium & Mono ants & Derived from genus name \\
\hline M. bifidum group & Northern fanged mono ants & Clypeus with prominent projections; restricted to north-western Australia \\
\hline M. carinatum group & Angled mono ants & Trunk often with distinct dorsal and lateral faces \\
\hline M. insolescens group & Monsoonal mono ants & Restricted to the monsoonal zone \\
\hline M. laeve group & Yellow mono ants & Colour yellowish \\
\hline M. longiceps group & Mallee mono ants & Characteristic of mallee habitats of southern semi-arid zone \\
\hline M. nigrius group & Black mono ants & Colour blackish \\
\hline M. fieldi & Hairy black mono ant & Hairs unusually dense and long \\
\hline M. nigrius & Little black mono ant & Very small \\
\hline M. rothsteini group & Smiling mono ants & Clypeal margin sinuate, giving it a smiling appearance \\
\hline M. whitei group & Southern fanged mono ants & $\begin{array}{l}\text { Clypeus with prominent projections; restricted to semi-arid southern } \\
\text { Australia }\end{array}$ \\
\hline Orectognathus & Goblin ants & Morphologically bizarre dacetines \\
\hline Pheidole & Big-headed ants & Major workers with disproportionately large heads \\
\hline P. hartmeyri group & Harvester big-headed ants & Large, specialist granivores of southern arid zone \\
\hline P. impressiceps & Giant big-headed ant & Very large size \\
\hline P. longiceps group & Knobbed big-headed ants & Pronotal shoulders with tubercles \\
\hline P. mjobergi group & Savanna big-headed ants & Major group in monsoonal region \\
\hline$P$. group A (Andersen 2000) & Speckled big-headed ants & Head densely punctate \\
\hline Podomyrma & Muscleman tree-ants & $\begin{array}{l}\text { Arboreal ants, with swollen femora that are reminiscent of 'muscle-man' } \\
\text { biceps }\end{array}$ \\
\hline P. adelaidae & Desert muscleman tree-ant & Only species in central Australia \\
\hline Pristomyrmex & Spiny jungle ants & With pronotal and propodeal spines; restricted to rainforest \\
\hline Pyramica & Detritus ants & $\begin{array}{l}\text { As in the related Strumigenys, tiny, cryptic dacetines with the appearance } \\
\text { of detritus }\end{array}$ \\
\hline Solenopsis & Thief ants & $\begin{array}{l}\text { Established common name for the only sub-genus (Diplorhoptrum) native } \\
\text { to Australia; known to steal food from nests of other ants (Shattuck 1999) }\end{array}$ \\
\hline Strumigenys & Snappy detritus ants & See Pyramica; elongate, trap-jaw mandibles \\
\hline Tetramorium & Pennant ants & Pennant-like appendage on sting \\
\hline T. impressum group & Impressive pennant ants & From species-group name \\
\hline T. laticephalum group & Harvester pennant ants & Seed-harvesters \\
\hline T. sjostedti group & Giant pennant ants & Largest of Australian Tetramorium \\
\hline T. spininode group & Royal pennant ants & $\begin{array}{l}\text { Described as the 'most spectacular' of the world's Tetramorium (Bolton } \\
\text { 1977); gaster with a crown-like flange }\end{array}$ \\
\hline T. striolatum group & Common pennant ants & Australia's most common species-group \\
\hline \multicolumn{3}{|l|}{ Subfamily Nothomyrmeciinae } \\
\hline Nothomyrmecia macrops & Dinosaur ant & Established common name, as considered a 'living fossil' (Taylor 1978) \\
\hline \multicolumn{3}{|l|}{ Subfamily Ponerinae } \\
\hline Amblyopone & Michelin ants & 'Fat-waisted' (petiole with broad attachment to gaster) \\
\hline
\end{tabular}




\begin{tabular}{|c|c|c|}
\hline Scientific name & Common name & Explanation \\
\hline A. australis & Southern michelin ant & Most conspicuous species in southern Australia \\
\hline Anochetus & Lesser snappy ants & Trap-jaw mandibles (as in related but larger Odontomachus) \\
\hline Bothroponera & Foaming ants & Venom with foaming substance \\
\hline B. dentata group & Toothed foaming ants & Dorso-posterior margin of petiole serially dentate \\
\hline B. excavata group & Eared foaming ants & Dorso-posterior margin of petiole with lateral projections \\
\hline B. porcata group & Striped foaming ants & First gastric segment conspicuously striate \\
\hline B. sublaevis group & Smooth foaming ants & Without gastric sculpture \\
\hline Diacamma & Bladder ants & $\begin{array}{l}\text { Possessing unique, bladder-like glands that regulate reproductive } \\
\text { dominance (Peeters \& Higashi 1989) }\end{array}$ \\
\hline D. australe & Australian bladder ant & Endemic to Australia \\
\hline D. levis & Smooth bladder ant & Less heavily sculptured than D. australe \\
\hline Discothyrea & Clubbed trigger ants & $\begin{array}{l}\text { Along with Proceratium, having a peculiarly reflexed, trigger-like gaster; } \\
\text { antennal club massively swollen }\end{array}$ \\
\hline Heteroponera & False pony ants & Related to Rhytidoponera \\
\hline Hypoponera & Crypt ants & Subterranean, cryptic habits \\
\hline Leptogenys & Genial killer ants & Specialist predators; 'genial' derived from 'genys' \\
\hline Odontomachus & Giant snappy ants & See Anochetus \\
\hline Platythyrea & Broad-nosed killer ants & Specialist predators with widely spaced frontal carinae \\
\hline$P$. parallela group & Northern broad-nosed killer ants & Tropical distribution \\
\hline P. turneri group & Southern broad-nosed killer ants & Southern distribution \\
\hline Onychomyrma & False army ants & Exhibit army ant-like behaviour \\
\hline Ponera & Blind crypt ants & Closely related to Hypoponera, but eyes usually absent \\
\hline Proceratium & Trigger ants & See Discothyrea \\
\hline Rhytidoponera & Pony ants & Genus name sounds like 'ride a pony' \\
\hline$R$. araneoides group & Spider pony ants & From species-group name \\
\hline R. aspera group & Rough blue pony ants & With strikingly blue iridescence; 'rough' from species-group name \\
\hline R. aurata group & Lesser-horned pony ants & Occipital corners produced into sharp angles (see related taurus group) \\
\hline R. convexa group & Convex pony ants & From species-group name \\
\hline R. impressa group & Blue pony ants & Most species with strikingly blue iridescence \\
\hline R. mayri group & Titan pony ants & Extremely large species \\
\hline R. metallica group & Metallic pony ants & From species-group name \\
\hline R. punctata group & Speckled pony ants & Integument punctate \\
\hline R. reticulata group & Netted pony ants & From species-group name \\
\hline R. taurus group & Greater-horned pony ants & $\begin{array}{l}\text { Occipital corners produced into horn-like projections (see related aurata } \\
\text { group) }\end{array}$ \\
\hline R. tenuis group & Delicate pony ants & Body small and slim \\
\hline R. turneri group & Shark-finned pony ants & Propodeum reflexed \\
\hline R. tyloxys group & Killer pony ants & Appear to be specialist predators \\
\hline \multicolumn{3}{|c|}{ Subfamily Pseudomyrmecinae } \\
\hline Tetraponera & Black tree ants & Black, arboreal ants \\
\hline T. nitida & Toothed black tree-ant & Small ventral teeth on petiole \\
\hline T. punctulata & Savanna black tree-ant & Occurs throughout monsoonal Australia \\
\hline
\end{tabular}

